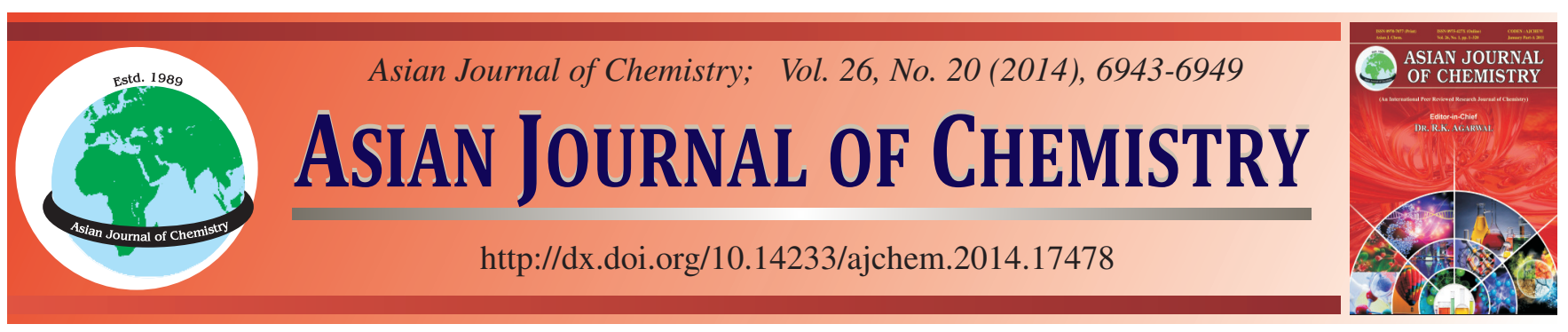

\title{
Hemisynthesis and Spectroscopic Characterization of Two Novel $O$-Allylated Benzophenones from Garcinia punctata Oliv. (Clusiaceae)
}

\author{
B. Ngameni ${ }^{1, *}$, G.W. Fotso ${ }^{2}$, E. NGachussi ${ }^{2}$, H.M. Poumale Poumale ${ }^{2}$, B.T. NGadjul ${ }^{2}$, Y. Shiono ${ }^{3}$ and T. Murayama ${ }^{3, *}$
}

${ }^{1}$ Department of Pharmacognosy and Pharmaceutical Chemistry, Faculty of Medicine and Biomedical Science, University of Yaounde I, P.O. Box. 8664, Yaounde, Cameroon

${ }^{2}$ Department of Organic Chemistry, Faculty of Science, University of Yaounde-1, P.O. Box. 812, Yaoundé, Cameroon

${ }^{3}$ Department of Bioresource Engineering, Faculty of Agriculture, Tsuruoka, Yamagata 997-8555, Japan

*Corresponding author: E-mail: bath_ngameni@yahoo.fr; mtetsuya@tr.yamagata-u.ac.jp

Four known natural compounds i.e., (-) isogarcinol, (+) 7-epigarcinol, stigmasterol and $\beta$-sitosterol-3-O- $\beta$-D-glucoside were isolated from the stem bark of Garcinia punctata oliv. From (-) isogarcinol, two new hemisynthesis $O$-allylated benzophenones were synthesized using allylbromide in the presence of potassium carbonate in anhydrous acetone. The structural elucidation of all the compounds was done by mass spectrometry, IR, 1D- and 2D-NMR analysis and also by comparison with previous reports. This is the first study to report on the one step allylation of the natural (-) isogarcinol using the base catalysis conditions.

Keywords: Garcinia punctata, Clusiaceae, (-) Isogarcinol, Allylation reaction, $O$-Allylated benzophenones.

\section{INTRODUCTION}

Natural product chemistry is an important area of research in organic chemistry. Compounds obtained from natural sources have been found to have a wide range of properties from anticancer activity to local anaesthetics. Other multitude uses have been reported from flavourings to dyes ${ }^{1}$. Scientists continuously screen plants, particularly of ethno-botanical significance, for their complex spectrum of biological and medicinal activities, which range from antibiotic to antitumor properties. Polyprenylated acylphloroglucinol derivatives, with a highly oxygenated bicyclo [3.3.1]nonane-2,4,9-trione or bicyclo [3.2.1] octane-2,4,8-trione core substituted with one or more prenyl or geranyl side chains, have been shown to occur in the plant family Clusiaceae ${ }^{2}$. Many compounds of this type have been identified from different plants in Garcinia ${ }^{2}$. This genus is native to Asia, Southern Africa and Polynesia ${ }^{3}$. Garcinia kola Heckel, commercially known as 'bitter cola', is a highly valued ingredient in African traditional medicine. The plant is cultivated throughout West Africa for its edible fruit and seeds. Seeds of Garcinia kola have been commonly used in folk medicine as aphrodisiac and general antidotes ${ }^{4}$. Garcinia species have been shown to be of pharmaceutical importance because recent studies showed that they can produce potent reverse transcriptase inhibitors of human immunodeficiency virus type $1^{5}$. The chemical constituents of this genus have also been reported to possess a wide range of biological activities, such as antimicrobial, antidepressant, anti-HIV, antitumor, antioxidant and cytotoxic effects ${ }^{6,7}$. The most extensively studied of these compounds is garcinol, which can induce apoptosis and inhibit cell survival and proliferation pathways such as MAPK and PI3K/Akt ${ }^{8}$. Garcinia punctata Oliv. is a tree distributed in the West and Southern parts of Cameroon. The stem bark is used in folk medicine as antidotes (venomous stings, bites, etc.), pain-killers and for the treatment of pulmonary disorders ${ }^{9}$. The powder from the bark of the tree is also used for the treatment of snake-bite in Congo (Brazzaville) and bark decoction is also used to treat cough and chest pain. Its chemical constituents have not yet been fully investigated. There are very limited studies on hemisynthetic reactions reported on this taxon. As part of a program to screen derivatives of major metabolites isolated from Garcinia species, we undertook the allylation of (-) isogarcinol (1) with allylbromide under basic conditions ${ }^{10}$. The aim of the current synthetic study was to provide an easy access to allylated benzophenones at $\mathrm{C}$-13 with two $\alpha, \beta$-unsaturated carbonyls and a carbonyl of a ketone in order to assess the potential pharmacological properties of the resulting synthetic products. In the present study, we report the isolation of two new hemisynthesis $O$-allylated Benzophenone derivatives 3 and 4 (Fig. 1) and four known natural compounds, (-) isogarcinol $(\mathbf{1})^{11},(+) 7$-epigarcinol $(2)^{12}$, stigmasterol $5^{13}$ and $\beta$-sitosterol- 
<smiles>CC(C)=CC[C@H]1C[C@]23C[C@@H](CC=C(C)C)[C@@H](C)[C@@]2(CC=C(C)C)C(=O)C(C(=O)c2ccc(O)c(O)c2)=C3OC1(C)C</smiles>

1<smiles>C=C(C)C(CC=C(C)C)CC12C[C@H](CC=C(C)C)[C@@H](C)[C@]1(CC=C(C)C)C(O)=C(C(=O)c1ccc(O)c(O)c1)C2=O</smiles>

2



3

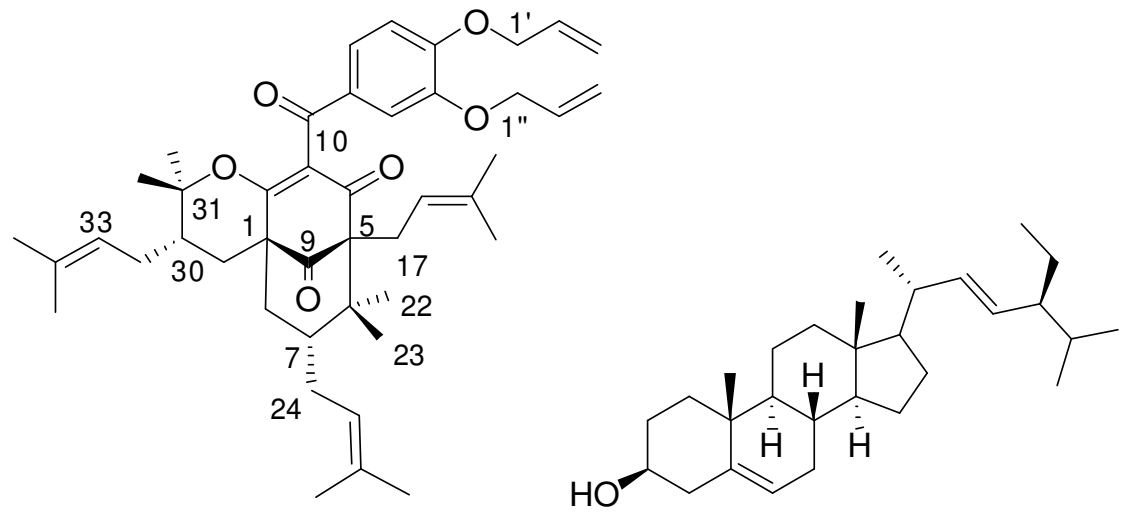

4
5

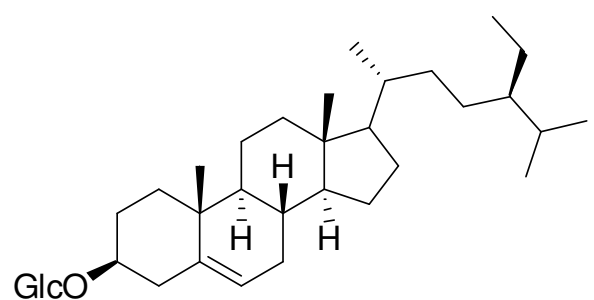

6

Fig. 1. Structure of the natural isolated compounds (1-2 and 5-6) and hemi synthetic (3-4)

3-O- $\beta$-D-glucoside $\mathbf{6}^{14}$ from the stem bark of $G$. punctata oliv. Their structures were identified on the basis of mass spectrometry, IR spectral and by interpretation of their spectroscopic data $\left({ }^{1} \mathrm{H},{ }^{13} \mathrm{C}\right.$ NMR, COSY, NOESY, HMQC and $\mathrm{HMBC}$ ) as well as comparison with those reported in the literature $^{11-14}$.

\section{EXPERIMENTAL}

General procedures. Optical rotations were measured on a Horiba- High Sensitive Polarimeter- SEPA-300. IR spectra were recorded on a Horiba-Fourier Transform Infra Red Spectrometer FT-710. NMR spectra were recorded on a EXSpectrometer- Joel-JNM-EX 400-FTNMR System. ESI-MS were recorded on a Micromass Quattro LC mass spectrometer. Thin layer chromatography (TLC) was carried out using Merck silica gel $60 \mathrm{~F}-254$ plates (layer thickness $0.25 \mathrm{~mm}$ ) and all solvents were distilled prior to use.

The stem bark of Garcinia punctata was collected in July 2007 at Kribi, in the southern region of Cameroon and identified by Mr. Victor Nana of the National Herbarium in Yaoundé, Cameroon where a voucher specimen (No. 30738 HNC) was deposited.

Extraction and isolation: The air-dried and powdered stem bark of $G$. punctata $(1 \mathrm{~kg})$ was macerated in either a mixture of $\mathrm{CH}_{2} \mathrm{Cl}_{2} / \mathrm{MeOH}$ (1:1) or in $\mathrm{MeOH}$ for 24 and $2 \mathrm{~h}$, respectively, at room temperature. The removal of solvent under reduced pressure yielded $45 \mathrm{~g}$ of brown extract. A mass of $40 \mathrm{~g}$ of this organic extract was submitted to flash liquid chromatography on silica gel $60(220 \mathrm{~g})$ and eluted with hexane-ethyl acetate (EtOAc) mixtures: (3:1), (1:1), (1:3) and finally with pure EtOAc to give 40 fractions of $250 \mathrm{~mL}$ each. Fractions were monitored by TLC and fractions showing similar ${ }^{1} \mathrm{H}$ NMR spectra were combined. Fractions 1-20 (10 g), obtained with $(3: 1 \mathrm{v} / \mathrm{v})$ hexane-EtOAc were combined and rechromatographed to column chromatography over silica gel using hexane-EtOAc as eluent with a continuous gradient (95:5 to $7: 3 \mathrm{v} / \mathrm{v})$ to give in the order of elution stigmasterol $(\mathbf{5}, 19.6$ $\mathrm{mg})$ and (-) isogarcinol $(\mathbf{1}, 835.8 \mathrm{mg})$. The major fractions 21-40 (25 g), obtained with (1:1 and 1:3 v/v) hexane-EtOAc mixture, respectively, were subjected to repeated column chromatography over silica gel using $\mathrm{CHCl}_{3}-\mathrm{MeOH}$ as eluent with a continuous gradient $(98: 2$ to $85: 15 \mathrm{v} / \mathrm{v})$ to yield: (-) isogarcinol (1, $124.1 \mathrm{mg}),(+)$ 7-epigarcinol (2, $45.4 \mathrm{mg})$ and $\beta$-sitosterol-3-O- $\beta$-D-glucoside $(\mathbf{6}, 44.2 \mathrm{mg})$.

Chemical and materials. allylation of (-) isogarcinol (1): To $80.4 \mathrm{mg}(0.13 \mathrm{mmol})$ of (-) isogarcinol (1) in dry acetone (8 mL), was added $\mathrm{K}_{2} \mathrm{CO}_{3}(0.3 \mathrm{~g})$ followed by allylbromide $(0.12 \mathrm{~mL}, \mathrm{~d}=1.43,0.17 \mathrm{~g}, 1.43 \mathrm{mmol})$. The reaction mixture was heated at $40{ }^{\circ} \mathrm{C}$ under reflux for $23 \mathrm{~h}$. At the end of the reaction, the solvent was evaporated under reduced pressure and the residue was diluted in water $(40 \mathrm{~mL} \times 3)$. The aqueous mixture was extracted with ethyl acetate $(3 \times 60 \mathrm{~mL})$ and the 
extract was dried by anhydrous $\mathrm{Na}_{2} \mathrm{SO}_{4}$. After evaporation of the solvent and purification by column chromatography on silica gel by elution with hexane- EtOAc system of increasing polarity, products $3\left(38.2 \mathrm{mg}, 0.06 \mathrm{mmol}, 48 \%, \mathrm{R}_{\mathrm{f}}\right.$ 0.24 , silica gel, hexane-EtOAc, 8:2 v/v) and 4 (25.3 mg, 0.04 mmol, $32 \%, \mathrm{R}_{\mathrm{f}} 0.45$, silica gel, hexane-EtOAc, 8:2 v/v) were obtained.

(-)-14- $\boldsymbol{O}$-Allylisogarcinol (3): Colourless oil; $[\alpha]_{D^{-}} 119.2^{\circ}$ $\left(c=1, \mathrm{CH}_{3} \mathrm{COCH}_{3}\right)$; IR $\left(\mathrm{CHCl}_{3}\right)\left(\mathrm{KBr}, v_{\max }, \mathrm{cm}^{-1}\right)$ : 3546-3442, 3082, 2975-2875, 2728, 2370, 2260, 1728, 1676, 1606, 1508, 1452, 1371-1346, 1279, 1188, 1122, 1090, 993-927, 773-715; ${ }^{1} \mathrm{H}-\mathrm{NMR}\left(\mathrm{C}_{5} \mathrm{H}_{5} \mathrm{~N}-d_{5}, 400 \mathrm{MHz}\right)\left(\right.$ Table-2), ${ }^{13} \mathrm{C} \mathrm{NMR}\left(\mathrm{C}_{5} \mathrm{H}_{5} \mathrm{~N}-\right.$ $d_{5}, 100 \mathrm{MHz}$ ) (Table-2); ESI-MS m/z (rel. int.): $643[\mathrm{M}+\mathrm{H}]^{+}$ (100, calcd. for $\left.\mathrm{C}_{41} \mathrm{H}_{54} \mathrm{O}_{6}, 642.3920\right)$; Elemental analysis: Calcd for $\mathrm{C}_{41} \mathrm{H}_{54} \mathrm{O}_{6}$ : C,76.60; $\mathrm{H}, 8.47$; O,14.93. Found: C,76.67; H,8.45; O, 14.87. COSY, HMQC and HMBC (Table-2 and Fig. 2).

(-)-13,14-Di- $\boldsymbol{O}$-allylisogarcinol (4): Colourless oil; $[\alpha]_{\mathrm{D}}$ - 448.1 ${ }^{\circ}\left(\mathrm{c}=1, \mathrm{CH}_{3} \mathrm{COCH}_{3}\right)$; IR $\left(\mathrm{CHCl}_{3}\right)\left(\mathrm{KBr}, \mathrm{v}_{\max }, \mathrm{cm}^{-1}\right)$ :3467-3400, 3292-3232, 2974-2858, 2497, 2366, 2324, 19921909, 1868-1837, 1793-1729, 1676-1604, 1508, 1458, 1437, 1375, 1277, 1182, 1124, 1092, 993, 949, 823, 766; ${ }^{1} \mathrm{H}$ NMR $\left(\mathrm{C}_{5} \mathrm{H}_{5} \mathrm{~N}-d_{5}, 400 \mathrm{MHz}\right)\left(\right.$ Table-3), ${ }^{13} \mathrm{C}$ NMR $\left(\mathrm{C}_{5} \mathrm{H}_{5} \mathrm{~N}-d_{5}, 100\right.$ $\mathrm{MHz}$ (Table-3); ESI-MS m/z (rel. int.): $683[\mathrm{M}+\mathrm{H}]^{+}(100$, calcd. for $\mathrm{C}_{44} \mathrm{H}_{58} \mathrm{O}_{6}, 682.4233$ ); Elemental analysis: Calcd for $\mathrm{C}_{44} \mathrm{H}_{58} \mathrm{O}_{6}$ : C, 77.38; H, 8.56; O, 14.06. Found: C,77.45; H, 8.48; O, 14.02. COSY, HMQC and HMBC (Table-3 and Fig. 2).

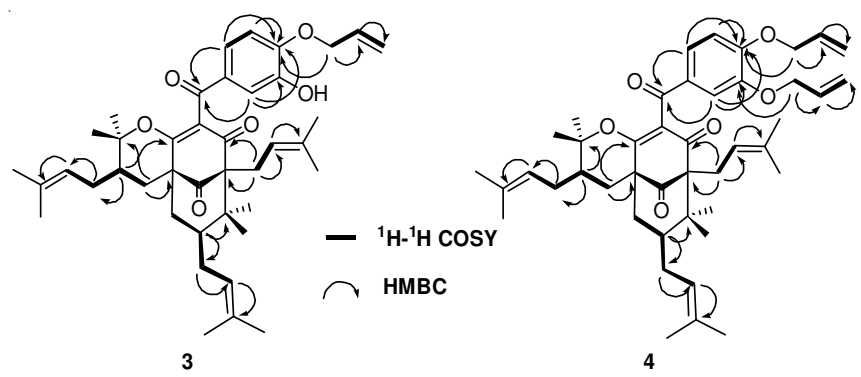

Fig. 2. Selected ${ }^{1} \mathrm{H}-{ }^{1} \mathrm{H}$ COSY and HMBC correlations in compounds (3) and (4), respectively

\section{RESULTS AND DISCUSSION}

Compound 1 was isolated as a brown powder. The molecular formula $\mathrm{C}_{38} \mathrm{H}_{50} \mathrm{O}_{6}$ was deduced by HREIMS from the ion peak at $m / z, 602.3686$. The ${ }^{13} \mathrm{C}$ NMR spectra of compound 1 showed the signals of two $\alpha, \beta$-unsaturated carbonyls and a carbonyl of a ketone respectively at $\delta 192,194$ and 206.9. The above data coupled with the presence on the ${ }^{13} \mathrm{C}$ NMR spectrum of two quaternary carbon signals at $\delta 68.1$ and $\delta 45.9$ were consistent with the presence of a polycyclic polyprenylated acylphloroglucinol (PPAP) structure ${ }^{11-12}$. The ${ }^{1} \mathrm{H}-\mathrm{NMR}$ spectra showed the presence of a 1,3,4-trisubstituted benzene ring with three aromatic protons appearing as an $\mathrm{ABC}$ system at $\delta 8.04$ $(1 \mathrm{H}, \mathrm{d}, J 2.0 \mathrm{~Hz}, \mathrm{H}-12), \delta 7.24(1 \mathrm{H}, \mathrm{d}, J 8.0 \mathrm{~Hz}, \mathrm{H}-15)$ and $\delta$ $7.63(1 \mathrm{H}, \mathrm{dd}, J 8.0,2.0 \mathrm{~Hz}, \mathrm{H}-16)$; ten methyl groups at $\delta 1.52$ (3H, s, Me-20), 1.70 (3H, s, Me-21), 1.00 (3H, s, Me-22), 1.25 (3 H, s, Me-23), 1.66 (3H, s, Me-27), 1.63 (3H,s, Me28). 1.19 (3 H, s, Me-32), 1.03 (3H, s, Me-33), 1.70 (3H, s, Me-37), 1.63 (3 H, s, Me-38) and vinylic protons of three prenyl groups respectively at $[\delta 5.04(1 \mathrm{H}$, brt, $J 6.5 \mathrm{~Hz}, \mathrm{H}-18)$;
$5.04(1 \mathrm{H}$, brt, $J 6.5 \mathrm{~Hz}, \mathrm{H}-25)$ and $5.37(1 \mathrm{H}$, brt, $J 6.5 \mathrm{~Hz}, \mathrm{H}-$ 35)]. The ${ }^{13} \mathrm{C}$ NMR and DEPT spectra of 1 exhibited 38 signals (Table-1), including 15 quaternary carbons in which two $\alpha, \beta$-unsaturated carbonyl carbons ( $\delta 192.0$ and 194.0) and a carbonyl of a ketone at $\delta$ 206.9, 8 methine, 5 methylene and 10 methyl carbon (Table-1). The axial orientation of the prenyl group in position 7 was deduced by the chemical shifts of the methyl groups C-22ax $(\delta 25.6)$ and C-23eq $(\delta 22.0)^{2,15,16}$. In the NOESY spectrum, nOe interactions between H-8ax, H-7 and Me-22ax ( $\delta 2.06,1.60$ and 1.00, respectively) confirmed the absolute configuration $(\mathrm{R})$ for $\mathrm{C}-7$. The absolute configuration of the bicycle [3.3.1] nonane with $\alpha \mathrm{C}(1)-\mathrm{C}(9)$ and $C(5)-C(9)$ bonds, was deduced from its negative $[\alpha]_{D}$ value $^{17,18}$. Compound 1 was then identified to (-) isogarcinol previously isolated from the root barks of Symphonia globulifera ${ }^{11}$.

The spectroscopic data of compound $\mathbf{2}$ were closely comparable to those of compound $\mathbf{1}$. However the ${ }^{1} \mathrm{H}$ NMR of this compound showed the presence of two additional olefinic protons of a methylene at $\delta 4.75$ (brs), 4.87 (brs) and only 9 methyl carbons instead of 10 in compound 1 suggesting the opening of the pyran ring. This information was confirmed by the analysis of the ${ }^{13} \mathrm{C}$ NMR and DEPT spectra of compound 2 which showed the presence of an olefinic carbon at $\delta 112$ (C-32) and the downfield chemical shifts of carbons C-2 and C-34 from $\delta 171.4$ to 189.1 and $\delta 29.3$ to 42.3 , respectively (Table-1). Compound 2 was then identified to (+)-7-epigarcinol previously isolated from the trunk latex of Moronobea coccinea $^{12}$.

In addition, two known compounds were also isolated i.e., stigmasterol $5^{13}$ and $\beta$-sitosterol-3-O- $\beta$-D-glucoside $\mathbf{6}^{14}$. The structures were identified by comparison with published data $^{13,14}$.

Benzophenones are a group of compounds bearing a 13carbon core that can be prenylated and/or further cyclized producing numerous structurally unique compounds ${ }^{19}$. Benzophenones have a limited natural distribution and are restricted to the Clusiaceae, Moraceae and a few other plant families ${ }^{19}$. Benzophenones are known to show various biological properties such as antioxidant, antimicrobial, antifungal, cytotoxic and anti-HIV activities ${ }^{19}$. We wish to further investigate the structure-activity relationship of allylic benzophenones observations by screening the $O$-allylated derivatives of (-) isogarcinol (1) synthesized in the present work. The onestep allylation of 1 under base reaction conditions using $\mathrm{K}_{2} \mathrm{CO}_{3}$ in dry acetone afforded the $O$-allylbenzophenone products $\mathbf{3}$ and $\mathbf{4}$ via the nucleophilic substitution between $\mathbf{1}$ and allylbromide (Scheme-I) ${ }^{10}$. The structural elucidation of these products was done by mass spectrometry, IR, 1D- and 2DNMR analysis.

Compound 3 or (-)-14- $O$-allylisogarcinol was isolated as a colourless oil in the mixture of hexane-EtOAc $(4: 1 \mathrm{v} / \mathrm{v})$. The compounds $\mathbf{1}$ and $\mathbf{3}$ shared several common spectral characteristics. However the ${ }^{1} \mathrm{H}-\mathrm{NMR}$ and ${ }^{13} \mathrm{C}$ NMR spectra of $\mathbf{3}$ showed the presence of an allyl group characterized by a doublet of two protons at $\left(\delta 4.61, \mathrm{~d}, J 5.2 \mathrm{~Hz}, \mathrm{H}-\mathbf{1}^{\prime}\right)$; a multiplet of an olefinic proton at $\delta 5.92$ and the two diastereotopic protons of a methylene group at $[\delta 5.12(\mathrm{brd}, J 10.4 \mathrm{~Hz})$ and $\delta$ 5.33 (brd, $J 17.4 \mathrm{~Hz}$ )]. This group was fixed in position 14 of 
TABLE-1

${ }^{1} \mathrm{H}(400 \mathrm{MHZ})$ AND ${ }^{13} \mathrm{C}$ NMR (100 MHZ) SPECTRAL DATA OF (-) ISOGARCINOL (1) AND (+) 7-EPI-GARCINOL (2) IN $\mathrm{C}_{5} \mathrm{H}_{5} \mathrm{~N}-d_{5}$

\begin{tabular}{|c|c|c|c|c|}
\hline \multirow{2}{*}{ Position } & \multicolumn{2}{|c|}{1} & \multicolumn{2}{|c|}{2} \\
\hline & $\delta_{\mathrm{H}}(m, J$ in $\mathrm{Hz})$ & $\delta_{\mathrm{C}}$ & $\delta_{\mathrm{H}}(m, J$ in $\mathrm{Hz})$ & $\delta_{\mathrm{C}}$ \\
\hline 1 & - & $51.1(\mathrm{~s})$ & - & $58.7(\mathrm{~s})$ \\
\hline 2 & - & $171.4(\mathrm{~s})$ & - & $189.1(\mathrm{~s})$ \\
\hline 3 & - & $127.2(\mathrm{~s})$ & - & $123.4(\mathrm{~s})$ \\
\hline 4 & - & 194.0 (s) & - & $194.7(\mathrm{~s})$ \\
\hline 5 & - & $68.1(\mathrm{~s})$ & - & $67.6(\mathrm{~s})$ \\
\hline 6 & - & $45.9(\mathrm{~s})$ & - & $48.2(\mathrm{~s})$ \\
\hline 7 & $1.59(\mathrm{dt}, 6.2,6.1)$ & 45.7 (d) & $1.50(\mathrm{~m})$ & $46.6(d)$ \\
\hline 8 & $\begin{array}{c}2.06(\mathrm{dd}, 14.1,7.3) \\
2.42(\mathrm{brd}, 14.1)\end{array}$ & $36.6(t)$ & $\begin{array}{l}2.10(\mathrm{~m}) \\
2.70(\mathrm{~m})\end{array}$ & $36.3(\mathrm{t})$ \\
\hline 9 & - & $206.9(\mathrm{~s})$ & - & $210.0(\mathrm{~s})$ \\
\hline 10 & - & $192.0(\mathrm{~s})$ & - & $191.7(\mathrm{~s})$ \\
\hline 11 & - & $129.8(\mathrm{~s})$ & - & $129.2(\mathrm{~s})$ \\
\hline 12 & $8.04(\mathrm{~d}, 2.0)$ & $115.5(\mathrm{~d})$ & $7.85(\mathrm{~d}, 1.6)$ & 116.7 (d) \\
\hline 13 & - & $146.7(\mathrm{~s})$ & - & $146.4(\mathrm{~s})$ \\
\hline 14 & - & $152.7(\mathrm{~s})$ & - & $152.7(\mathrm{~s})$ \\
\hline 15 & $7.24(\mathrm{~d}, 8.0)$ & $115.4(\mathrm{~d})$ & $7.18(\mathrm{~d}, 8.0)$ & $114.8(\mathrm{~d})$ \\
\hline 16 & $7.63(\mathrm{dd}, 8.0,2.0)$ & $123.2(\mathrm{~d})$ & $7.55(\mathrm{dd}, 8.0,1.6)$ & 124.1 (d) \\
\hline 17 & $\begin{array}{l}2.71(\mathrm{dd}, 13.6,5.2) \\
2.90(\mathrm{dd}, 13.6,8.0)\end{array}$ & $25.6(t)$ & $\begin{array}{c}2.45(\mathrm{brd}, 13.6) \\
2.86(\mathrm{brd}, 9.6)\end{array}$ & $25.9(\mathrm{t})$ \\
\hline 18 & 5.37 (brt, 6.5) & $120.6(d)$ & 5.53 (brt, 6.4) & 121.1 (d) \\
\hline 19 & - & $135.2(\mathrm{~s})$ & - & $133.5(\mathrm{~s})$ \\
\hline 20 & $1.52(\mathrm{~s})$ & $25.6(q)$ & $1.66(\mathrm{~s})$ & $25.7(q)$ \\
\hline 21 & $1.70(\mathrm{~s})$ & $17.7(\mathrm{q})$ & $1.62(\mathrm{~s})$ & $17.9(\mathrm{q})$ \\
\hline 22 & $1.00(\mathrm{~s})$ & $25.6(q)$ & $1.09(\mathrm{~s})$ & $26.6(q)$ \\
\hline 23 & $1.25(\mathrm{~s})$ & $22.0(q)$ & $1.34(\mathrm{~s})$ & $22.5(q)$ \\
\hline 24 & $\begin{array}{l}1.82(\mathrm{dd}, 14.2,9.5) \\
3.21(\mathrm{dd}, 14.4,9.5)\end{array}$ & $29.3(t)$ & - & $29.3(\mathrm{t})$ \\
\hline 25 & $5.04(\mathrm{brt}, 6.5)$ & $125.6(d)$ & 5.03 (brt, 6.4 ) & $125.1(\mathrm{~d})$ \\
\hline 26 & - & $134.7(\mathrm{~s})$ & - & $131.7(\mathrm{~s})$ \\
\hline 27 & $1.66(\mathrm{~s})$ & $25.4(q)$ & $1.66(\mathrm{~s})$ & $25.3(q)$ \\
\hline 28 & $1.63(\mathrm{~s})$ & $17.9(\mathrm{q})$ & $1.62(\mathrm{~s})$ & $17.9(\mathrm{q})$ \\
\hline 29 & $\begin{array}{c}1.14(\mathrm{dd}, 13.9,13.7) \\
3.27(\mathrm{dd}, 13.9,3.1)\end{array}$ & $28.0(\mathrm{t})$ & - & $32.5(\mathrm{t})$ \\
\hline 30 & $1.65(\mathrm{~m})$ & $42.7(\mathrm{~d})$ & - & $43.8(d)$ \\
\hline 31 & - & $86.1(\mathrm{~s})$ & - & $148.4(\mathrm{~s})$ \\
\hline 32 & $1.19(\mathrm{~s})$ & $20.6(q)$ & $\begin{array}{l}4.75 \text { (brs) } \\
4.87 \text { (brs) }\end{array}$ & $112.0(\mathrm{t})$ \\
\hline 33 & $1.03(\mathrm{~s})$ & $26.1(\mathrm{q})$ & $1.74(\mathrm{~s})$ & $25.5(q)$ \\
\hline 34 & $\begin{array}{l}1.96 \text { (brd, 14.1) } \\
2.42 \text { (brd, 14.1) }\end{array}$ & $29.3(t)$ & - & $42.3(t)$ \\
\hline 35 & 5.04 (brt, 6.5) & $122.4(\mathrm{~d})$ & 5.26 (brt, 6.4) & $123.3(\mathrm{~d})$ \\
\hline 36 & - & $135.0(\mathrm{~s})$ & - & \\
\hline 37 & $1.70(\mathrm{~s})$ & $25.1(q)$ & $1.81(\mathrm{~s})$ & $25.9(\mathrm{q})$ \\
\hline 38 & $1.63(\mathrm{~s})$ & $17.2(\mathrm{q})$ & $1.80(\mathrm{~s})$ & $17.9(\mathrm{q})$ \\
\hline
\end{tabular}

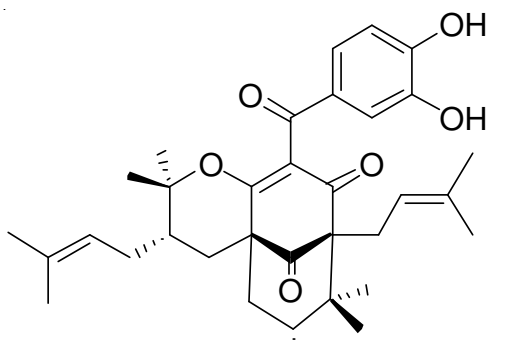

1<smiles>CCC=C(C)C</smiles>

1) $\mathrm{K}_{2} \mathrm{CO}_{3}$, dry acetone

2) $>\mathrm{Br}$

Heated $\sim 40{ }^{\circ} \mathrm{C}, 23 \mathrm{~h}$

3: $\mathrm{R}_{1}=\mathrm{H}$<smiles>[R]=C=CC[14CH2]</smiles>

4: $\mathrm{R}_{1}=\mathrm{R}_{2}=\gamma$

Scheme-I: allylation of (-) isogarcinol (1)

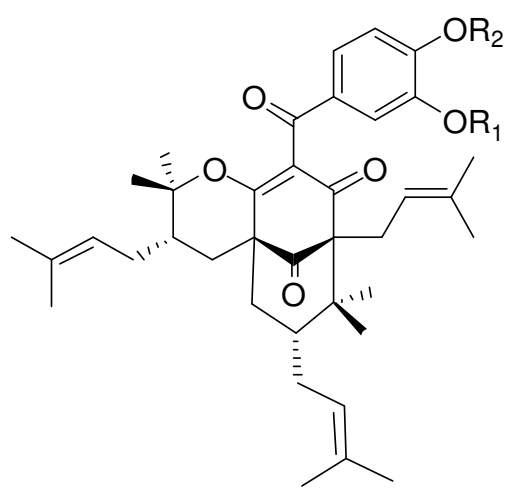


compound 1 by the HMBC correlation between the protons $\mathrm{H}-1^{\prime}$ at $\delta 4.61$ and the carbon $\mathrm{C}-14$ at $\delta 152.3$. This position was confirmed by the downfield shift of the ortho $\mathrm{C}-15$ from $\delta 115.4$ to $\delta 112.9$. Also, the NOESY spectra helps to support the position of the allyl group at $\mathrm{C}-14$ by the correlation between the methylene protons $\mathrm{H}-1^{\prime}$ at $\delta 4.61$ and the proton $\mathrm{H}-15$ of the benzene ring at $\delta 7.06$, respectively.

Compound 4 or (-)-13,14-di- $O$-allylisogarcinol was isolated as a colourless oil in the mixture of hexane-EtOAc $(9: 1 \mathrm{v} / \mathrm{v})$. Two allylic groups were characterized in the ${ }^{1} \mathrm{H}$ NMR and ${ }^{13} \mathrm{C}$ NMR spectra of 4 , respectively $\left[\delta 4.63\left(\mathrm{~d}, J 4.8, \mathrm{H}-1^{\prime}\right)\right.$; $\delta 6.01\left(\mathrm{~m}, \mathrm{H}-2^{\prime}\right) ; \delta 5.18$ ( $\left.\mathrm{m}, \mathrm{H}-3^{\prime} \mathrm{a}\right) ; \delta 5.39$ (m, H-3'b)] and $[\delta$
4.50 (brs, H-1"); $\delta 6.01$ (m, H-2"); $\delta 5.18$ ( m, H-3"a); $\delta 5.39$ $\left.\left(\mathrm{m}, \mathrm{H}-3^{\prime \prime} \mathrm{b}\right)\right]$. These allyl groups were fixed in position 13 and 14 of compound 1 due to the HMBC correlation between the protons $\mathrm{H}-1$ ' and $\mathrm{H}-1^{\prime \prime}$ and the carbons C-14 and C-13, respectively at $\delta 153.6$ and 149.0.

There are no allylation studies on natural (-) isogarcinol (1) reported in the literature. The allylated products $\mathbf{3}$ and $\mathbf{4}$ were isolated here for the first time via hemisynthesis by transformation of natural product $\mathbf{1}$ as a starting material. The structural identities of analogs $\mathbf{3}$ and $\mathbf{4}$ were fully established by analysis of spectral data, mainly IR, mass, ${ }^{1} \mathrm{H}$ - and ${ }^{13} \mathrm{C}$ NMR and 2D NMR (Fig. 2; Tables 2 and 3).

\begin{tabular}{|c|c|c|c|c|c|}
\hline \multicolumn{6}{|c|}{  } \\
\hline Position & $\delta_{\mathrm{H}}(\mathrm{m}, J$ in $\mathrm{Hz})$ & $\delta_{\mathrm{C}}$ & $\operatorname{COSY}\left({ }^{1} \mathrm{H}-{ }^{1} \mathrm{H}\right)$ & HMQC & $\begin{array}{c}\text { HMBC }{ }^{2} \mathrm{~J} \text { and }{ }^{3} \mathrm{~J} \text {-correlated } \\
\mathrm{C}^{1} \mathrm{H} \rightarrow{ }^{13} \mathrm{C}\end{array}$ \\
\hline 1 & - & $51.7(\mathrm{~s})$ & - & - & - \\
\hline 2 & - & 171.4 (s) & - & - & - \\
\hline 3 & - & $126.4(\mathrm{~s})$ & - & - & - \\
\hline 4 & - & $194.5(\mathrm{~s})$ & - & - & - \\
\hline 5 & - & $68.7(\mathrm{~s})$ & - & - & - \\
\hline 6 & - & $46.5(\mathrm{~s})$ & - & - & - \\
\hline 7 & $1.94(\mathrm{~m})$ & $46.3(\mathrm{~d})$ & 8,24 & 46.3 & $6,8,24,25$ \\
\hline 8 & $2.39(\mathrm{brd}, 14.4)$ & $39.4(t)$ & 7 & 39.4 & $1,7,9$ \\
\hline 9 & - & $207.4(\mathrm{~s})$ & - & - & - \\
\hline 10 & - & $192.9(\mathrm{~s})$ & - & - & - \\
\hline 11 & - & $131.9(\mathrm{~s})$ & - & - & - \\
\hline 12 & 7.95 (brs) & $116.6(d)$ & 16 & 116.6 & $10,11,13,14$ \\
\hline 13 & - & $148.4(\mathrm{~s})$ & - & - & - \\
\hline 14 & - & $152.3(\mathrm{~s})$ & - & - & - \\
\hline 15 & 7.06 (brd, 8.4) & $112.9(\mathrm{~d})$ & 16 & 112.9 & 13,14 \\
\hline 16 & 7.65 (brd, 8.4) & $122.3(d)$ & 15 & 122.3 & $10,11,14$ \\
\hline 17 & $\begin{array}{l}2.73(\mathrm{dd}, 13.6,4.8) \\
2.91(\mathrm{dd}, 13.6,6.4)\end{array}$ & $26.2(\mathrm{t})$ & 18 & 26.2 & $5,4,18$ \\
\hline 18 & 5.40 (brt, 6.4) & $121.2(\mathrm{~d})$ & 17 & 121.2 & $17,19,20,21$ \\
\hline 19 & - & $134.1(\mathrm{~s})$ & - & - & - \\
\hline 20 & $1.53(\mathrm{~s})$ & $26.1(q)$ & - & 26.1 & 18,19 \\
\hline 21 & $1.88(\mathrm{~s})$ & $18.3(\mathrm{q})$ & - & 18.3 & 18,19 \\
\hline 22 & $1.02(\mathrm{~s})$ & $26.2(\mathrm{q})$ & - & 26.2 & 5 \\
\hline 23 & $1.26(\mathrm{~s})$ & $21.2(\mathrm{q})$ & - & 21.2 & 5 \\
\hline 24 & $\begin{array}{l}1.82(\mathrm{dd}, 14.1,8.5) \\
2.26(\mathrm{dd}, 14.1,2.5)\end{array}$ & $29.9(\mathrm{t})$ & 7,25 & 29.9 & 7,25 \\
\hline 25 & $5.06(\mathrm{brt}, 6.2)$ & $125.9(\mathrm{~d})$ & 24 & 125.9 & $26,27,28$ \\
\hline 26 & - & $132.9(\mathrm{~s})$ & - & - & - \\
\hline 27 & $1.68(\mathrm{~s})$ & $25.7(q)$ & - & 25.7 & 25,26 \\
\hline 28 & $1.64(\mathrm{~s})$ & $18.5(\mathrm{q})$ & - & 18.5 & 25,26 \\
\hline 29 & $\begin{array}{l}1.21(\mathrm{dd}, 13.9,13.7) \\
3.28(\mathrm{dd}, 13.6,3.2)\end{array}$ & $28.6(t)$ & 30 & 28.6 & 1,2 \\
\hline 30 & $1.65(\mathrm{~m})$ & $43.4(d)$ & 29,34 & 43.4 & 31,35 \\
\hline 31 & - & $86.9(\mathrm{~s})$ & - & - & - \\
\hline 32 & $1.21(\mathrm{~s})$ & $20.7(q)$ & - & 20.7 & 31 \\
\hline 33 & $1.01(\mathrm{~s})$ & $26.2(q)$ & - & 26.2 & 31 \\
\hline 34 & $\begin{array}{l}1.79(\mathrm{~m}) \\
1.96(\mathrm{~m})\end{array}$ & $29.9(\mathrm{t})$ & 30,35 & 29.9 & 35,36 \\
\hline 35 & 5.06 (brt, 6.6) & $122.3(\mathrm{~d})$ & 34 & 122.3 & $34,36,38$ \\
\hline 36 & - & $133.3(\mathrm{~s})$ & - & - & - \\
\hline 37 & $1.71(\mathrm{~s})$ & $22.6(q)$ & - & 22.6 & 35,36 \\
\hline 38 & $1.61(\mathrm{~s})$ & $17.8(\mathrm{q})$ & - & 17.8 & 35,36 \\
\hline $1^{\prime}$ & $4.61(\mathrm{~d}, 5.2)$ & $69.8(\mathrm{t})$ & $2^{\prime}$ & 69.8 & $2^{\prime}, 3^{\prime}, 14$ \\
\hline $2^{\prime}$ & $5.92(\mathrm{~m})$ & $133.4(\mathrm{~d})$ & $1^{\prime}, 3^{\prime}$ & 133.4 & $1^{\prime}, 3^{\prime}$ \\
\hline $3^{\prime}$ & $\begin{array}{l}5.12(\text { brd, } 10.4) \\
5.33(\text { brd, } 17.2)\end{array}$ & $118.1(\mathrm{t})$ & $2^{\prime}$ & 118.1 & $1^{\prime}, 2^{\prime}$ \\
\hline
\end{tabular}




\begin{tabular}{|c|c|c|c|c|c|}
\hline \multicolumn{6}{|c|}{ 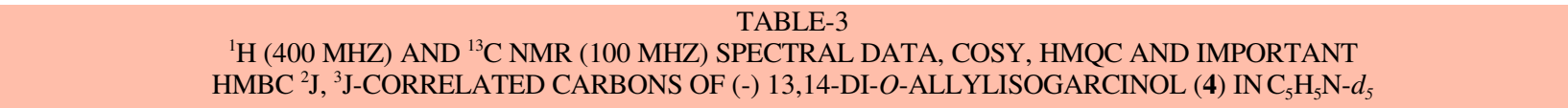 } \\
\hline Position & $\delta_{\mathrm{H}}(\mathrm{m}, J$ in $\mathrm{Hz})$ & $\delta_{\mathrm{C}}$ & $\operatorname{COSY}\left({ }^{1} \mathrm{H}-{ }^{1} \mathrm{H}\right)$ & HMQC & $\begin{array}{c}\text { HMBC }{ }^{2} \mathrm{~J} \text { and }{ }^{3} \mathrm{~J} \text {-correlated } \\
\mathrm{C}^{1} \mathrm{H} \rightarrow{ }^{13} \mathrm{C}\end{array}$ \\
\hline 1 & - & $51.7(\mathrm{~s})$ & - & - & - \\
\hline 2 & - & $171.6(\mathrm{~s})$ & - & - & - \\
\hline 3 & - & $126.1(\mathrm{~s})$ & - & - & - \\
\hline 4 & - & $194.4(\mathrm{~s})$ & - & - & - \\
\hline 5 & - & $68.8(\mathrm{~s})$ & - & - & - \\
\hline 6 & - & $46.5(\mathrm{~s})$ & - & - & - \\
\hline 7 & $1.81(\mathrm{~m})$ & $46.4(d)$ & 8,24 & 46.4 & $6,8,24,25$ \\
\hline 8 & $\begin{array}{c}2.09(\mathrm{dd}, 7.6 ; 14.4) \\
2.40(\mathrm{brd}, 14.4)\end{array}$ & $39.4(t)$ & 7 & 39.4 & $1,7,9$ \\
\hline 9 & - & $207.2(\mathrm{~s})$ & - & - & - \\
\hline 10 & - & $192.6(\mathrm{~s})$ & - & - & - \\
\hline 11 & - & $131.4(\mathrm{~s})$ & - & - & - \\
\hline 12 & 7.82 (brs) & $112.9(\mathrm{~d})$ & 16 & 112.9 & $10,11,13,14$ \\
\hline 13 & - & $149.0(\mathrm{~s})$ & - & - & - \\
\hline 14 & - & $153.6(\mathrm{~s})$ & - & - & - \\
\hline 15 & 7.06 (brd, 8.4) & $112.8(\mathrm{~d})$ & 16 & 112.8 & 13,14 \\
\hline 16 & $7.60(\mathrm{brd}, 8.4)$ & $122.3(\mathrm{~d})$ & 15 & 122.3 & $10,11,14$ \\
\hline 17 & $\begin{array}{l}2.70(\mathrm{~m}) \\
2.93(\mathrm{~m})\end{array}$ & $26.2(t)$ & 18 & 26.2 & $5,4,18$ \\
\hline 18 & 5.35 (brt, 6.5 ) & $121.2(\mathrm{~d})$ & 17 & 121.2 & $17,19,20,21$ \\
\hline 19 & - & $134.1(\mathrm{~s})$ & - & - & - \\
\hline 20 & $1.55(\mathrm{~s})$ & $26.2(\mathrm{q})$ & - & 26.2 & 18,19 \\
\hline 21 & $1.91(\mathrm{~s})$ & $18.3(\mathrm{q})$ & - & 18.3 & 18,19 \\
\hline 22 & $1.01(\mathrm{~s})$ & $26.3(q)$ & - & 26.3 & 5 \\
\hline 23 & $1.26(\mathrm{~s})$ & $22.6(q)$ & - & 22.6 & 5 \\
\hline 24 & - & $29.9(\mathrm{t})$ & 7,25 & 29.9 & 7,25 \\
\hline 25 & 5.04 (brs) & $125.9(\mathrm{~d})$ & 24 & 125.9 & $26,27,28$ \\
\hline 26 & - & $132.9(\mathrm{~s})$ & - & - & - \\
\hline 27 & $1.68(\mathrm{~s})$ & $26.1(q)$ & - & 26.1 & 25,26 \\
\hline 28 & $1.66(\mathrm{~s})$ & $18.5(\mathrm{q})$ & - & 18.5 & 25,26 \\
\hline 29 & $\begin{array}{l}1.08 \text { (brt, 13.6) } \\
3.26 \text { (brd, 10.8) }\end{array}$ & $28.6(t)$ & 30 & 28.6 & 1,2 \\
\hline 30 & $1.63(\mathrm{~m})$ & $43.4(d)$ & 29,34 & 43.4 & 31,35 \\
\hline 31 & - & $86.9(\mathrm{~s})$ & - & - & - \\
\hline 32 & $1.23(\mathrm{~s})$ & $21.2(q)$ & - & 21.2 & 31 \\
\hline 33 & $1.01(\mathrm{~s})$ & $26.7(q)$ & - & 26.7 & 31 \\
\hline 34 & $2.05(\mathrm{~m})$ & $29.9(\mathrm{t})$ & 30,35 & 29.9 & 35,36 \\
\hline 35 & 4.91 (brs) & $122.3(\mathrm{~d})$ & 34 & 122.3 & $34,36,38$ \\
\hline 36 & - & $133.4(\mathrm{~s})$ & - & - & - \\
\hline 37 & $1.73(\mathrm{~s})$ & $25.8(\mathrm{q})$ & - & 25.8 & 35,36 \\
\hline 38 & $1.58(\mathrm{~s})$ & $17.9(\mathrm{q})$ & - & 17.9 & 35,36 \\
\hline $1^{\prime}$ & $4.63(\mathrm{~d}, 4.8)$ & $69.7(\mathrm{t})$ & $2^{\prime}$ & 69.7 & $2^{\prime}, 3^{\prime}, 14$ \\
\hline $2^{\prime}$ & $6.01(\mathrm{~m})$ & $133.4(\mathrm{~d})$ & $1^{\prime}, 3^{\prime}$ & 133.4 & $1^{\prime}, 3^{\prime}$ \\
\hline $3^{\prime}$ & $\begin{array}{l}5.18(\mathrm{~m}) \\
5.39(\mathrm{~m})\end{array}$ & $117.7(\mathrm{t})$ & $2^{\prime}$ & 117.7 & $1^{\prime}, 2^{\prime}$ \\
\hline $1^{\prime \prime}$ & 4.50 (brs) & $69.6(t)$ & $2^{\prime \prime}$ & & $2^{\prime}, 3^{\prime \prime}, 13$ \\
\hline $2^{\prime \prime}$ & $6.01(\mathrm{~m})$ & $133.7(\mathrm{~d})$ & $1^{\prime \prime}, 3^{\prime \prime}$ & & $1^{\prime \prime}, 3^{\prime \prime}$ \\
\hline $3^{\prime \prime}$ & $\begin{array}{l}5.18(\mathrm{~m}) \\
5.39(\mathrm{~m})\end{array}$ & $117.5(\mathrm{t})$ & $2^{\prime \prime}$ & & $1^{\prime \prime}, 2^{\prime \prime}$ \\
\hline
\end{tabular}

\section{Conclusion}

In order to assess the importance of the double bond of allyl substituents and the hydroxyl groups of (-) isogarcinol (1) on its pharmacological properties, a convenient methodology of allylation was used for the preparation of two allylic benzophenones, namely, (-) 14-O-allylisogarcinol (3) and (-) 13,14-di- $\boldsymbol{O}$-allylisogarcinol (4). This study revealed for, the first time, the formation of a mono- and a di- $O$-allylicbenzo- phenones 3 and 4 (Scheme-I), whose natural occurrence has not been demonstrated previously. Interestingly, this synthesis showed that only a major compound (C-14-monoallylated benzophenone) and minor adduct (C-13,14-diallylated benzophenone) are formed. The $\mathrm{C}-13$ monoallylated benzophenone was not formed under the condition of this allylation, may be due to a long chain chelated hydroxyl group at C- 13 of isogarcinol (1). Specifically, only the non-chelated phenolic hydroxyl group at C-14 of $\mathbf{1}$ afforded preponderant monoallylated 
reaction adduct 3 with a high yield. It will be necessary to verify further the formation of C-13 monoallylated benzophenones under drastic conditions. Our study is in progress to optimize the yields of these allylated synthetic compounds and to evaluate their pharmacological activities in comparison with the natural product $\mathbf{1}$.

\section{ACKNOWLEDGEMENTS}

The authors BN and MT are grateful to the MATSUMAE for their financial support of this research and for a travel grant to the Department of Chemistry, University of Yamagata (Japan).Thanks are also due to Prof. Fokunang Charles for the critical technical review of the manuscript.

\section{REFERENCES}

1. P.S. Bailey and C.A. Bailey, Organic Chemistry, Prentice-Hall Inc, New Jersey, edn 5 (1995).

2. R. Ciochina and R.B. Grossman, Chem. Rev., 106, 3963 (2006)

3. Y.H. Li, Flora Reipublicae Popularis Sinicae, Science Press, Beijing, pp 89-110 (1990).

4. M.M. Iwu, A.D. Diop, L. Meserole and O.C. Okunji, Ethnomed. Drug Discovery, 1, 191 (2002)

5. K.R. Gustafson, J.W. Blunt, M.H.G. Munro, R.W. Fuller, T.C. McKee, J.H. Cardellina II, J.B. McMahon, G.M. Cragg and M.R. Boyd, Tetrahedron, 48, 10093 (1992).

6. S.X. Huang, C. Feng, Y. Zhou, G. Xu, Q.B. Han, C.F. Qiao, D.C. Chang, K.Q. Luo and H.X. Xu, J. Nat. Prod., 72, 130 (2009).
7. M. Masullo, C. Bassarello, H. Suzuki, C. Pizza and S.J. Piacente, Agric. Food Chem., 56, 5205 (2008).

8. J. Hong, S.J. Kwon, S. Sang, J. Ju, J.N. Zhou, C.T. Ho, M.T. Huang and C.S. Yang, Free Radic. Biol. Med., 42, 1211 (2007).

9. H.M. Burkill, The Useful Plants of West Tropical Africa, Families E-I. Royal Botanic Gardens Kew, edn 2. Vol. 2, pp. ISBN No. 0-94764356-7 (1994).

10. S. Mukherjee, V. Kumar, A.K. Prasad, H.G. Raj, M.E. Bracke, C.E. Olsen, S.C. Jain and V.S. Parmar, Bioorg. Med. Chem., 9, 337 (2001).

11. G. Marti, V. Eparvier, C. Moretti, S. Prado, P. Grellier, N. Hue, O. Thoison, B. Delpech, F. Guéritte and M. Litaudon, Phytochemistry, 71, 964 (2010).

12. G. Marti, V. Eparvier, C. Moretti, S. Susplugas, S. Prado, P. Grellier, P. Retailleau, F. Guéritte and M. Litaudon, Phytochemistry, 70, 75 (2009).

13. V. Rukachaisirikul, W. Kaewnok, S. Koysomboon, S. Phongpaichit and W.C. Taylor, Tetrahedron, 56, 8539 (2000).

14. E. Elfita, M. Muharni, M. Latief, D. Darwati, A. Widiyantoro, S. Supriyatna, H.H. Bahti, D. Dachriyanus, P. Cos, L. Maes, K. Foubert, S. Apers and L. Pieters, Phytochemistry, 70, 907 (2009).

15. W. Hamed, S. Brajeul, F. Mahuteau-Betzer, O. Thoison, S. Mons, B. Delpech, N.V. Hung, T. Sevenet and C. Marazano, J. Nat. Prod., 69, 774 (2006).

16. A.L. Piccinelli, O. Cuesta-Rubio, M.B. Chica, N. Mahmood, B. Pagano, M. Pavone, V. Barone and L. Rastrelli, Tetrahedron, 61, 8206 (2005).

17. M. Iinuma, H. Tosa, T. Tanaka, S. Kanamaru, F. Asai, Y. Kobayashi, K. Miyauchi and R. Shimano, Biol. Pharm. Bull., 19, 311 (1996).

18. N. Krishnamurthy, Y.S. Lewis and B. Ravindranath, Tetrahedron Lett., 22, 793 (1981).

19. S. Baggett, E.P. Mazzola and E.K. Kennelly, Studies Nat. Prod. Chem., 32, 721 (2005). 